\title{
Design and Test of a Mobile Antenna System With Tri-Band Operation for Broadband Satellite Communications and DBS Reception
}

\author{
Soon Young Eom, Member, IEEE, Seong Ho Son, Young Bae Jung, Soon Ik Jeon, Sergei A. Ganin, \\ Anatoly G. Shubov, Alexey K. Tobolev, and Alexander V. Shishlov, Member, IEEE
}

\begin{abstract}
The tri-band mobile antenna system for broadband multimedia services in the $K a / K$-band and a simultaneous direct broadcast service in the $K u$-band has been developed. The radiating part of the antenna consists of a shaped dual reflector system and a tri-band feed of circular polarization. The low-profile offset main reflector has an aperture size of $60 \mathrm{~cm} \times 20 \mathrm{~cm}$; therefore, a fan beam is formed which is sharp in azimuth and wide in elevation. The feed system contains a protruding dielectric rod for $\mathrm{Ka} / \mathrm{K}$-band and $2 \times 2$ active phased array. The latter provides the beam for $K u$-band along with four fast electrical beams for stable satellite tracking. The new tracking algorithm based on a fusion technique involving various sensors and fast electrical beams has also been introduced. The antenna system was mounted on a large ship and a test car, and successfully operated via Koreasat-3.
\end{abstract}

Index Terms-Dual reflector, mobile antenna system, satellite tracking, tri-band feed.

\section{INTRODUCTION}

I N RECENT years, the demand for wireless communication services has advanced from fixed narrow bands to mobile broadband services. The capability of a mobile antenna system should provide wireless internet services, wireless LAN and multimedia services, as well as high quality broadcasting services simultaneously on moving vehicles via $K a / K u$-band geo-stationary satellites. Reflector antennas with mechanical beam steering occupy an important place in the area of mobile communication systems [1]-[5]. They make possible high gain and an admissible side lobe level at minimum cost. Recently, a shaped dual reflector antenna with an elongated main reflector and a circular sub-reflector was utilized in several projects (for example in [1], [2]). It has a low profile design along with a high gain fan beam. Also, due to an almost circular sub-reflector aperture, a feed with axial symmetry operating at both a linear and a circular polarization and providing a low cross-polarization can be used. At the same time, the aforementioned antennas have an "on-set" sub-reflector located in the central part of the main

Manuscript received November 7, 2006; revised July 1, 2007.

S. Y. Eom, Y. B. Jung, and S. I. Jeon are with the Radio Technology Group, Electronics and Telecommunications Research Institute (ETRI), Daejeon, 305700, Korea (e-mail: syeom@etri.re.kr).

S. H. Son is with the Department of Mechanical Engineering, Pohang University of Science and Technology (POSTECH), Pohang, 790-784, Korea.

S. A. Ganin, A. G. Shubov, A. K. Tobolev and A. V. Shishlov are with APEX Joint Stock Company, 107066 Moscow, Russia.

Color versions of one or more of the figures in this paper are available online at http://ieeexplore.ieee.org.

Digital Object Identifier 10.1109/TAP.2007.908819 reflector aperture that creates a blockage effect. Furthermore, these antennas operate in the $K u$-band only.

In this paper, a tri-band dual-reflector antenna with high directivity and electrical satellite tracking for operation via the geostationary satellite Koreasat-3, and incorporating $\mathrm{Ka} / \mathrm{K} \mathrm{com}$ munication and $K u$ broadcasting transponders, is described. The antenna has been developed at the Electronics and Telecommunications Research Institute (ETRI) and can be mounted on cars, ships, trains, and airplanes to provide broadband satellite multimedia and broadcasting services. We have considered a dual-reflector antenna on the basis of both on-set and off-set configurations which can explain the blockage effect of a main reflector aperture by a sub-reflector. The on-set structure enables a lower positioning of the antenna, but it engenders a severe aperture blockage which generates a high level of side lobes in the vertical plane, thereby causing gain degradation. However, the off-set structure without a blockage gives the best electrical performances, but brings bulkier and higher antenna dimensions. Therefore, an off-set scheme with a partial blockage which can provide a reasonable height and side lobe level has been chosen for the purposes of this paper.

Many of the published studies on multiband feeds show that the majority of technical decisions mainly boil down to two principles. First, a common horn or a common waveguide is used, and an excitation of each frequency band is effected through appropriate slots or probes. Second, several radiators with multichannels are foreseen. The internal channel corresponds to the highest frequency band, whereas a peripheral channel or a set of channels are used for lower frequency bands. The choice of a feed type depends on the relationship, or spacing, between the operating frequency bands. If the spacing is not more than 2-3 times, it is preferable to employ the single-horn scheme. Otherwise, it is better to use separate radiators for different frequency bands.

In our case, the feed should provide not only a multiband operation with frequency spacing of around 3 times, but must have a tracking function. The tracking capability of a reflector antenna can be achieved to some extent by displacing a feed from the reflector focal point. It can provide an angular beam tilted from the bore-site direction. There are several approaches to tracking systems. In radars and communication systems with very narrow antenna beams, monopulse feeds are usually used for this purpose [6]. They can be realized on the basis of multihorn schemes or multimode, single-horn schemes. All these modifications permit the measurement of RF signals simultaneously from several beams. It is superfluous in communication systems with 
TABLE I

REQUIRED DESIGN SPECIFICATIONS

\begin{tabular}{|c|c|c|c|}
\hline Classification & \multicolumn{3}{|c|}{ Specifications } \\
\hline Frequency band [GHz] & $\begin{array}{c}\text { Ka-band (Tx) } \\
30.085-30.885\end{array}$ & $\begin{array}{c}\text { K-band (Rx 1) } \\
20.355-21.155\end{array}$ & $\begin{array}{c}\text { Ku-band (Rx 2) } \\
11.7-12.0\end{array}$ \\
\hline Transmission EIRP [dBW] & 43.0 Min. & - & - \\
\hline Reception G/T [dB/K] & - & 12.0 & 7.5 \\
\hline Antenna gain [dBi] & 39.0 & 36.0 & 29.5 \\
\hline Polarization & RHCP & LHCP & LHCP \\
\hline Side lobe level & \multicolumn{4}{|c}{ Meet ITU-R S.465-5 (Transmission) } \\
\hline
\end{tabular}

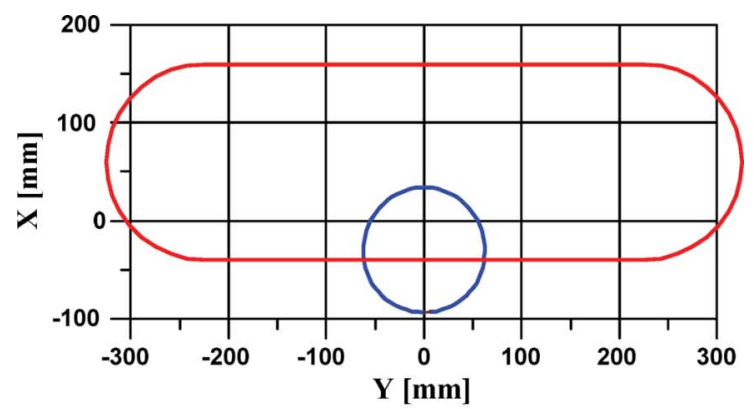

(a)

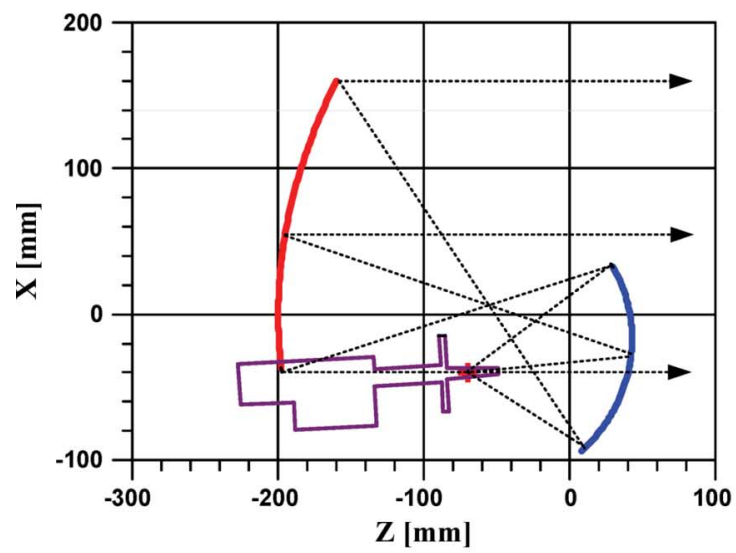

(b)

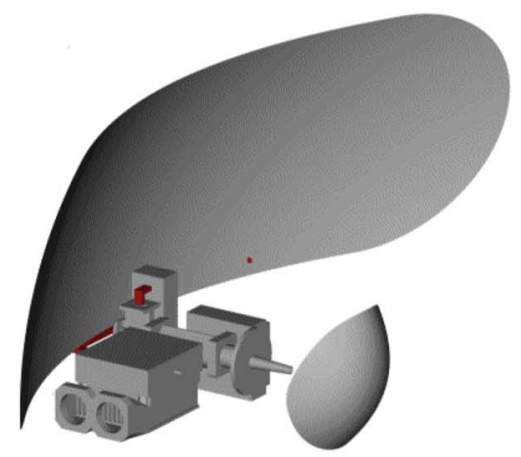

(c)

Fig. 1. Scheme of the radiating active channel module: (a) front-view, (b) sideview, and (c) 3-D overview.

wider beams where a simpler scheme using conical scanning or sequential lobbing can be applied. It is known that four discrete sequential beam positions are sufficient for realizing an antenna's tracking capability. Here, we have chosen the electrical tracking method with sequential beam lobbing. In combination with other open-loop angular sensors it provides sufficient accuracy for the beam pointing required in the antenna mounted on an agile

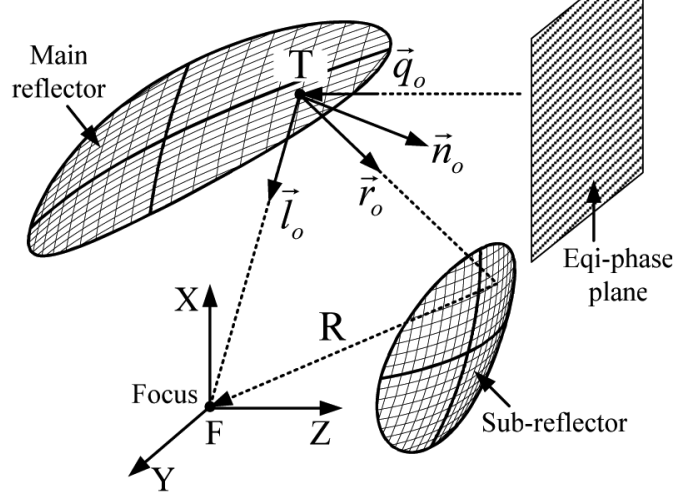

Fig. 2. Dual reflector antenna structure.

vehicle for communications. A new optimal tracking algorithm has been developed, and this is introduced in Section II-C. A prototype antenna was fabricated and both its indoor and outdoor tests have been performed and described in this paper.

\section{TRI-BAnd Mobile AnTEnNA System Design}

The tri-band mobile antenna system should meet the specifications presented in Table I. The antenna comprises a Radiating Active Channel Module (RACM), Beam Forming Module (BFM), Frequency Converter Module (FCM), Satellite Tracking Module (STM), and a Power Supply Module (PSM). However, only the details of the design of the RACM, BFM and STM, which are the core modules, will be described in this paper.

\section{A. Radiating Active Channel Module}

The RACM consists of a shaped dual-reflector, a tri-band feed with a dual-band polarizer and an orthomode transducer (OMT), a $K a$-band high power amplifier, and a $K$-band low noise amplifier with a built-in microstrip $\mathrm{Rx}$ band pass filter. The feed radiator comprises two parts; a dielectric rod for the $\mathrm{Ka} / \mathrm{K}$-band and four patch elements operated in the $K u$-band. The scheme of the RACM to show the arrangement of the tri-band feed and the RF blocks is shown in Fig. 1. Ku-band low noise amplifiers are included in the BFM, which is considered in Section II-B below.

The dual-reflector antenna geometry is shown in Fig. 2. It is designed through two steps. In the first step, the feed type and its parameters as well as the initial geometry of the dualreflector antenna (reflector size, initial reflector shape, location of the feed) are determined. In the second step, a shaping of dual-reflector surfaces is performed. 
Gregorian type offset geometry is chosen in the antenna structure because it is more compact and has a lower cross-polarization in comparison with Cassegrain geometry. The initial reflector surface is obtained by a geometrical optics (GO) approach [7]. The design simulation for the tri-band feed is performed by using a commercial HFSS simulator. The radiation pattern simulation and the shaping of the reflector surface are based on the use of physical optics (PO) according to the design procedure described in [8].

In the GO approach, the surfaces of the dual-reflector are formed to provide a uniform phase in the reflector aperture. Because the ratio of the major axis to the minor axis of the reflector aperture should be large enough for a low profile antenna, the elliptic paraboloidal surface of the main reflector according to the formula (1) is chosen

$$
Z=\frac{X^{2}}{4 F_{X}}+\frac{Y^{2}}{4 F_{Y}}
$$

The sub-reflector has a parametrically described surface which is formed as an ensemble of intersections of GO rays reflected from the main reflector being illuminated by a plane wave with a family of ellipsoids; one focus of the ellipsoid coincides with the phase center of the feed, whereas the other focus of the ellipsoid is placed on the main reflector from the view point of the GO-ray reflection. In this case, a circular aperture contour of the sub-reflector can be determined by formulas (2a), (2b) which represent Fermat's principle and (3), which describes the reflection law

$$
\begin{aligned}
R(T) & =\frac{P(T)}{1-e(T)\left(\overrightarrow{l_{o}}(T), \overrightarrow{r_{o}}(T)\right)} \\
P(T) & =\frac{\left(S_{o}-S(T)\right)^{2}+l(T)^{2}}{2\left(S_{o}-S(T)\right)} \\
\overrightarrow{r_{o}}(T) & =\overrightarrow{q_{o}}-2\left(\vec{n}(T), \overrightarrow{q_{o}}\right)
\end{aligned}
$$

where $T$ is a point on the main reflector, $R(T)$ is the distance from the focal point $F$ to sub-reflector along the ray, $P(T)$ is the focusing parameter of the ellipsoid, $\vec{n}$ is the unit vector perpendicular to the main reflector surface, $\overrightarrow{q_{o}}$ is the unit vector of a plane wave ray coming to the point $T$ on the main reflector, (it is perpendicular to the equiphase plane wave front), $\overrightarrow{l_{o}}$ is the vector directed to the focus from the main reflector, $\overrightarrow{r_{o}}$ is the vector of the GO ray reflected from the point $T$ on the main reflector, $S_{0}$ is the optical length from the antenna focus $F$ to the equiphase plane, $l(T)$ is the distance from the focus $F$ to the reflector point $T$, and $S(T)$ is the optical length from the point $T$ to the equiphase plane. The geometry of the antenna is determined by the focal lengths $F_{x}$ and $F_{y}$, by the dimensions of the main reflector $D_{x}$ and $D_{y}$, by the mutual position of the feed and the sub-reflector as defined by $P(T)$, and by the optical length from the focus $F$ to the equiphase plane.

The synthesis of the initial reflector surfaces is performed for the off-set dual-reflector structure with a partial blockage, as shown in Fig 1(a). The main reflector has an oval rim. The ratio of the oval major axis to its minor axis is $3.25: 1$. The dimensions of the main reflector are $650 \mathrm{~mm}$ (max. depth $130 \mathrm{~mm}$ ) for the major axis and $200 \mathrm{~mm}$ (max. depth $15 \mathrm{~mm}$ ) for the minor-axis,

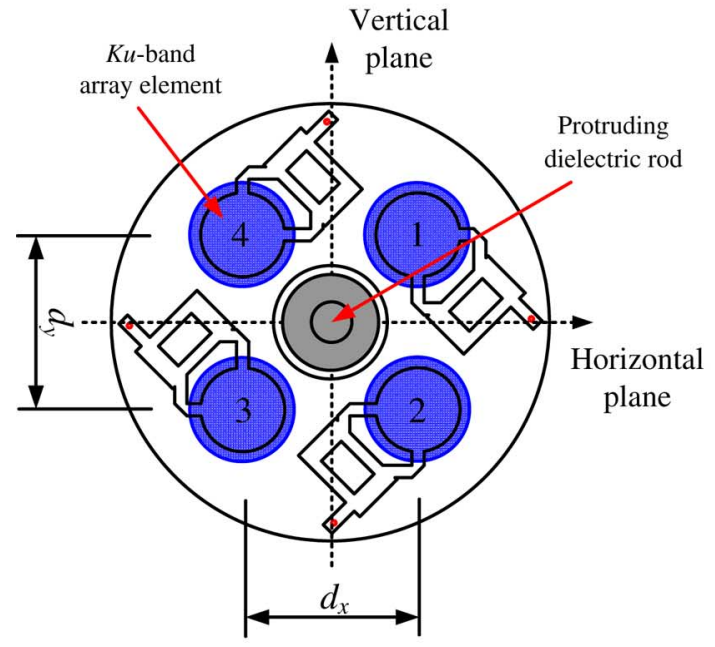

(a)

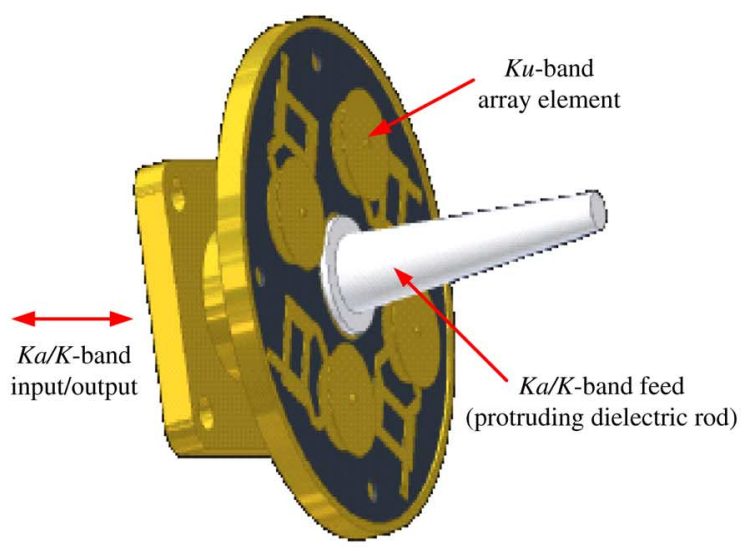

(b)

Fig. 3. Tri-band feed: (a) top-view and (b) side-view.

while the sub-reflector has a circular aperture with a diameter of $116 \mathrm{~mm}$ and maximum depths of $21 \mathrm{~mm}$ (vertical axis) and $44 \mathrm{~mm}$ (horizontal axis). The blockage of the reflector aperture by the sub-reflector causes antenna gain degradation of about $0.3 \mathrm{~dB}$. As shown in Fig. 1(b), the feed is located near the focal point, which is positioned at about $100 \mathrm{~mm}$ from the center of the sub-reflector. The phase center of the $K a / K$-band part of the feed is placed directly to the focal point $F$, and the $K u$-band array elements are placed $20 \mathrm{~mm}$ back from this focal point. The antenna gain performances simulated at the $K a / K$-band were simultaneously optimized at this position.

Once the GO synthesis is finished, the dual-reflector surfaces are represented as the form of a two-dimensional cubic spline with variable nodes. The shaping of its surfaces is performed in order to maximize the antenna gain as well as to meet the international radiation regulation of the antenna transmission pattern ITU-R S.465-5 [9]. Through this additional optimization of the performances simulated by the $\mathrm{PO}$ approach, antenna gain increments of more than $0.5 \mathrm{~dB}$ are obtained in the $\mathrm{Ka}$ - and $K$-band, respectively. Because the level of the $K u$-band DBS signal served from Koreasat- 3 is rather high, the geometry of the reflectors optimized at the $\mathrm{Ka} / \mathrm{K}$-band is also acceptable for operation in the $K u$-band. This permits the use of a Ku-band 
TABLE II

Simulated Performances of the Four Squint Partial Beams

\begin{tabular}{|c|c|c|c|c|c|c|}
\hline \multirow{2}{*}{$\begin{array}{c}\text { Partial } \\
\text { beam No. }\end{array}$} & \multirow{2}{*}{$\begin{array}{l}\text { Array elements } \\
\text { forming } \\
\text { a partial beam }\end{array}$} & \multicolumn{2}{|c|}{ Beam offset angle [deg] } & \multicolumn{2}{|c|}{ Gain [dBi] } & \multirow{2}{*}{$\begin{array}{c}\text { Relative } \\
\text { gain loss } \\
\text { [dB] }\end{array}$} \\
\hline & & Azimuth & Elevation & $\begin{array}{l}\text { Antenna } \\
\text { bore-site }\end{array}$ & \begin{tabular}{|c|} 
Partial \\
beam direction
\end{tabular} & \\
\hline 1 & Elements 1,2 & +0.85 & 0.0 & 27.3 & 29.3 & -2.0 \\
\hline 2 & Elements 2, 3 & 0.0 & -1.8 & 27.3 & 28.2 & -0.9 \\
\hline 3 & Elements 3,4 & -0.85 & 0.0 & 27.2 & 29.2 & -2.0 \\
\hline 4 & Elements 1,4 & 0.0 & +2.2 & 27.2 & 28.3 & -1.1 \\
\hline
\end{tabular}

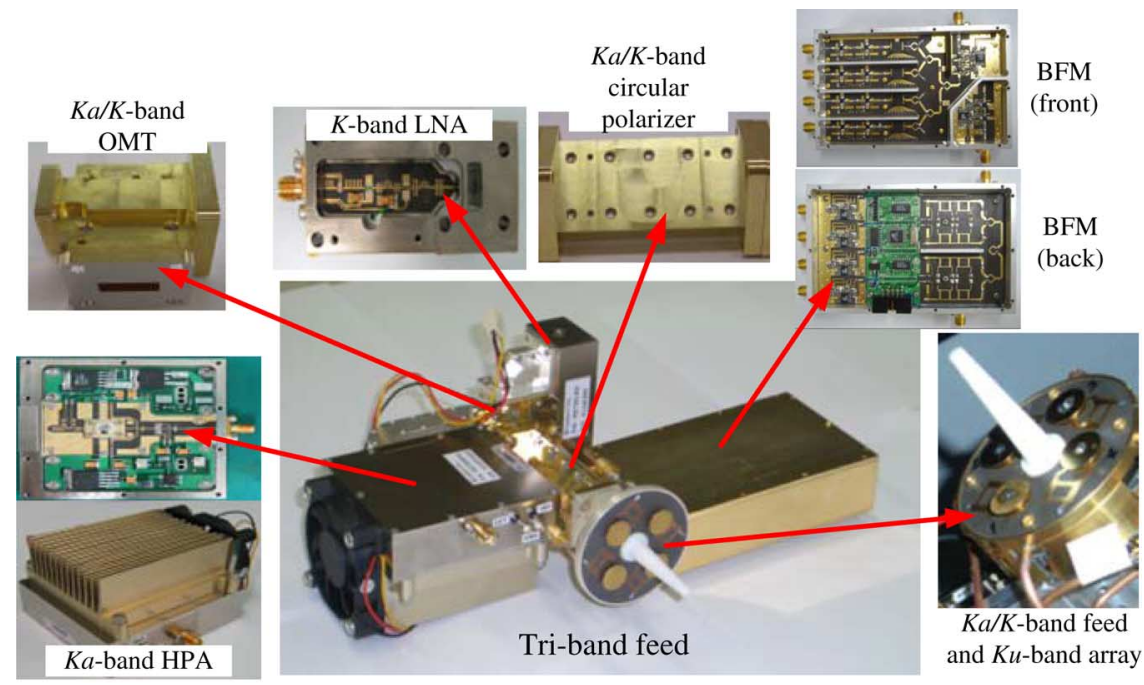

Fig. 4. Assembly and components of the tri-band feed.

signal coming from the satellite for broadcasting as well as for satellite tracking.

The feed proposed for the tri-band operation in this paper is shown in Fig. 3. It comprises a $\mathrm{Ka} / \mathrm{K}$ dual-band protruding dielectric rod surrounded by $K u$-band patch elements of a $2 \times 2$ array with optimal element spacing. Note, if a scalar corrugated horn is used in this feed for the $\mathrm{Ka} / \mathrm{K}$-band, it would be impossible to realize the tri-band feed in view of the large size of the aperture of the corrugated horn and hence the excessively large spacing between the $K u$-band array elements. Such a spacing of elements causes low antenna efficiency and high side lobe level. Therefore, the protruding dielectric rod is used in order to efficiently radiate electromagnetic power towards the sub-reflector. The $K u$-band part of the feed is a planar structure, while the $K a / K$-band part is a longitudinal one. It can be supposed that this design provides low mutual coupling between the $K u$-band port and the $K a$-band port. Practically, the isolation measured between them is more than $35 \mathrm{~dB}$ within the $\mathrm{Ka}$ operating band.

The $K a / K$-band radiator is a dielectric rod with a stepped transition, which is fed by the circular waveguide with a diameter of $10.67 \mathrm{~mm}$ that is connected with the $\mathrm{Ka} / \mathrm{K}$ dual-band circular polarizer, and the $K a / K$ orthomode transducer (OMT). The radiator is made of Teflon with relative permittivity $\varepsilon_{r}=2.05$. To ensure the required performances of the radiator pattern in the $\mathrm{Ka} / \mathrm{K}$-band, the shape and aperture dimensions of the dielectric rod are optimized. Consequently, a rod length of $35 \mathrm{~mm}$ and a tip tapering to $4 \mathrm{~mm}$ in diameter are chosen. Only two orthogonal main modes $\mathrm{TE}_{11}$ can be excited into the circular waveguide at the $K$-band. However, at the $K a$-band, additional, higher modes such as $\mathrm{TM}_{10}$ mode and two $\mathrm{TE}_{21}$ modes can exist in the circular waveguide. These modes can be also excited from the $\mathrm{Ka} / \mathrm{K}$-band radiating structure. The simulation on the feed including the polarizer and OMT using HFSS has shown that excited higher mode levels do not exceed $-25 \mathrm{dBc}$ for the mode $\mathrm{TM}_{10}$ and $-35 \mathrm{dBc}$ for the mode $\mathrm{TE}_{21}$.

Four array elements of the $K u$-band feed are arranged at a $45^{\circ}$ offset from the $\mathrm{x}-\mathrm{y}$ axes, as shown in Fig. 3. Considering the operational bandwidth, a microstrip stack structure [10] was adapted. Each radiator consists of a fed element measuring $9.6 \mathrm{~mm}$ in diameter, implemented on soft Teflon of relative permittivity $\varepsilon_{\mathrm{r}}=2.17$ and dielectric thickness of $h=0.508 \mathrm{~mm}$, with a parasitic conductive disc of $11.0 \mathrm{~mm}$ in diameter placed $2 \mathrm{~mm}$ above the fed element. Both the fed element and the parasitic disk are fixed by a metallic support penetrating through the center of each; it is possible that this technique does not affect the electrical performance including the radiation pattern in the operating band. A $90^{\circ}$ branch line hybrid coupler is used to form a circular polarization. In addition, four array elements are rotationally arranged at $90^{\circ}$ intervals to decrease the cross polarization of the $K u$-band sum beam. The spacing between the array elements is determined by considering the element patterns while taking into account their mutual coupling. Also, the element spacing must be small enough to suppress grating lobes of the array factor. When taking into account these factors, the values $d_{x}=d_{y}=0.8 \lambda_{\mathrm{o}}$ are chosen, where $\lambda_{\mathrm{o}}$ is a wavelength at a central frequency of the $K u$-band.

To form the $K u$-band sum beam, signals coming from four radiators are added. In the tracking channel, the right-squint par- 
tial beam (No. 1) and the left-squint partial beam (No. 3) are formed by each pair composed of array elements 1, 2 and 3, 4 respectively. The amplitudes between the two beams are compared in order to find the azimuth direction to a satellite from a bore-site. Similarly, to find the elevation direction, the amplitudes between the down-squint partial beam (No. 2) and the upper-squint partial beam (No. 4) formed by array elements 2,3 and 1, 4 respectively are compared. Table II shows the electrical performances simulated on the four squint partial beams.

The dual-band polarizer is used to generate two orthogonal circular polarizations according to the incident ports in the $\mathrm{Ka} / \mathrm{K}$-band. The feed is composed of the two half-housings of a circular waveguide and two comb plates which are inserted between them. Because of its simplicity, absence of any tuning elements, and effective performance, it is suitable for many applications in the millimeter-band [11]. The electrical performance of the comb polarizer is optimized by varying the radius of the circular waveguide as well as the number and geometric parameters of the comb cogs. The measured return losses of the polarizer show good match performances; less than $-18.8 \mathrm{~dB}$ in the $K$-band and less than $-26.4 \mathrm{~dB}$ in the $K a$-band; the cross-polarization levels measured in the $K a / K$-band are less than $-30.0 \mathrm{~dB}$ and $-22.9 \mathrm{~dB}$, respectively.

The OMT separates or combines two $K a$ - and $K$-band signals with independently orthogonal linear polarizations, and has a stepped structure inside for impedance matching. The designed OMT shows quite suitable electrical performances. The input and output return losses measured in the $K a / K$-band are less than $-32 \mathrm{~dB}$ and $-22 \mathrm{~dB}$ respectively, while the insertion losses are less than 0.08 and $0.05 \mathrm{~dB}$ respectively. The isolation between the Tx and Rx ports is more than $42 \mathrm{~dB}$.

The $K a$-band high-power amplifier (HPA) provides output power amplification of the transmitting signal. It is supplied with a detector of transmitting power, a stable dc supply, and a cooler to protect the HPA from overheating. The HPA should provide $\mathrm{P}_{1 \mathrm{dBc}}$ output power of more than $2.5 \mathrm{~W}$ in order to meet the required Tx EIRP. To this end, a TGA $49054 \mathrm{~W}(33 \mathrm{~dB}$ of linear gain) device from Triquint is used at the output end. A signal detector circuit with a coupling of about $-20 \mathrm{~dB}$ is adapted in the output circuit to monitor the transmission signal. The measured $\mathrm{P}_{1 \mathrm{dBc}}$ output power of the HPA is more than $34.1 \mathrm{dBm}$ in the operating band, and the detected voltage at the $\mathrm{P}_{1 \mathrm{dBc}}$ operating point is approximately $2.0 \mathrm{~V}$.

The $K$-band low noise amplifier (LNA) should have a noise figure of less than $2.5 \mathrm{~dB}$ in order to meet the required $\mathrm{G} / \mathrm{T}$ characteristic. The CHA2090 (noise figure $2.0 \mathrm{~dB}$, linear gain $23 \mathrm{~dB}$ ) from UMS is used in the first stage of the LNA. The input part of the LNA includes a built-in Rx-band pass filter designed with microstrip parallel stubs in order to reject the $\mathrm{Ka}$-band signal. By introducing this kind of input filter, the noise figure is degraded by about $0.3 \mathrm{~dB}$. The measured noise figure of the LNA is less than $2.4 \mathrm{~dB}$ within the operating band, and its linear gain is more than $34.9 \mathrm{~dB}$.

The overall effective equivalent noise temperature $T_{\text {eff } K}$ [12], [13] in the $K$-band, which includes an antenna noise temperature being referenced at the LNA input, can be estimated by summing the external antenna temperature at $45^{\circ}$ elevation angle $(26.7 \mathrm{~K})$, internal antenna noise temperature due to active

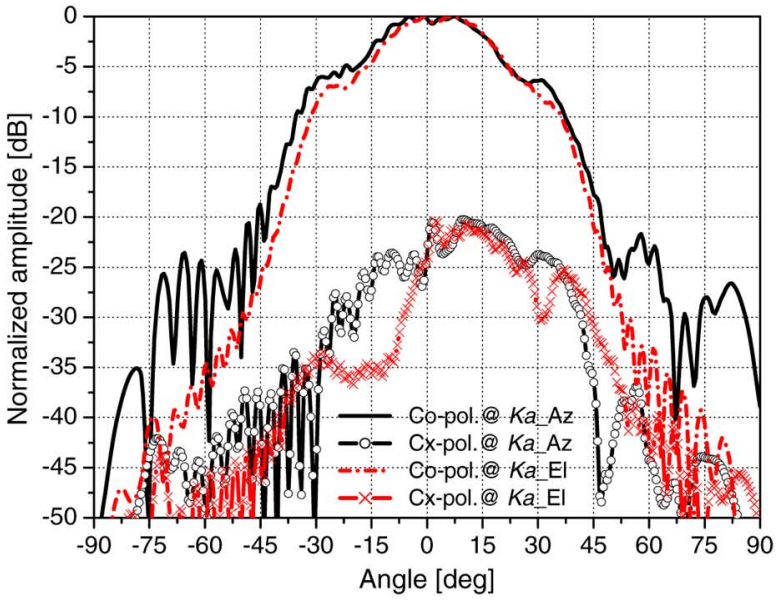

(a)

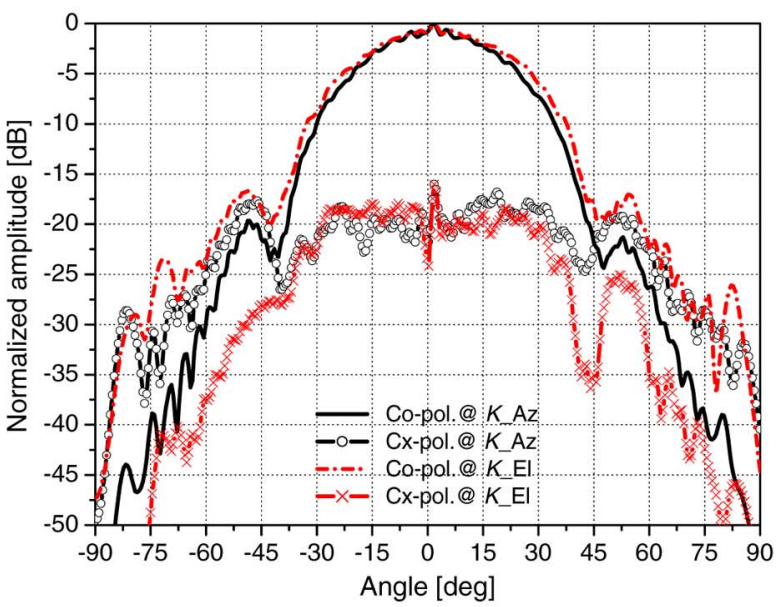

(b)

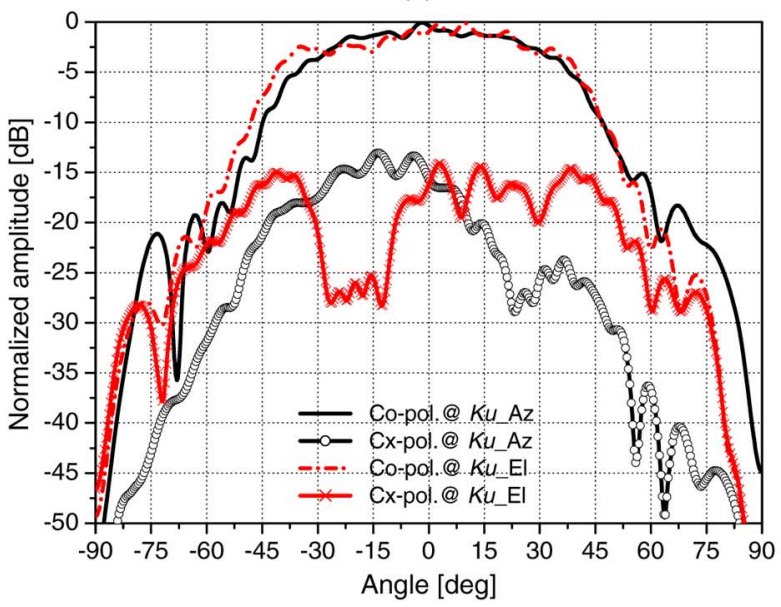

(c)

Fig. 5. Measured radiation patterns of the tri-band feed: (a) $K a$-band ( $\mathrm{f}=$ $30.485 \mathrm{GHz})$, (b) $K$-band $(\mathrm{f}=20.755 \mathrm{GHz}$ ), and (c) $K u$-band (single element, $\mathrm{f}=11.85 \mathrm{GHz}$ )

loss $(31.9 \mathrm{~K})$ and LNA noise temperature (214 K). Therefore, the estimation of system noise temperature $T_{\text {eff } K}$ in the $K$-band gives the value of $273 \mathrm{~K}$.

Photos of the fabricated tri-band feed are shown in Fig. 4, and the radiation pattern characteristics of the tri-band feed measured in the anechoic chamber are shown in Fig. 5. 


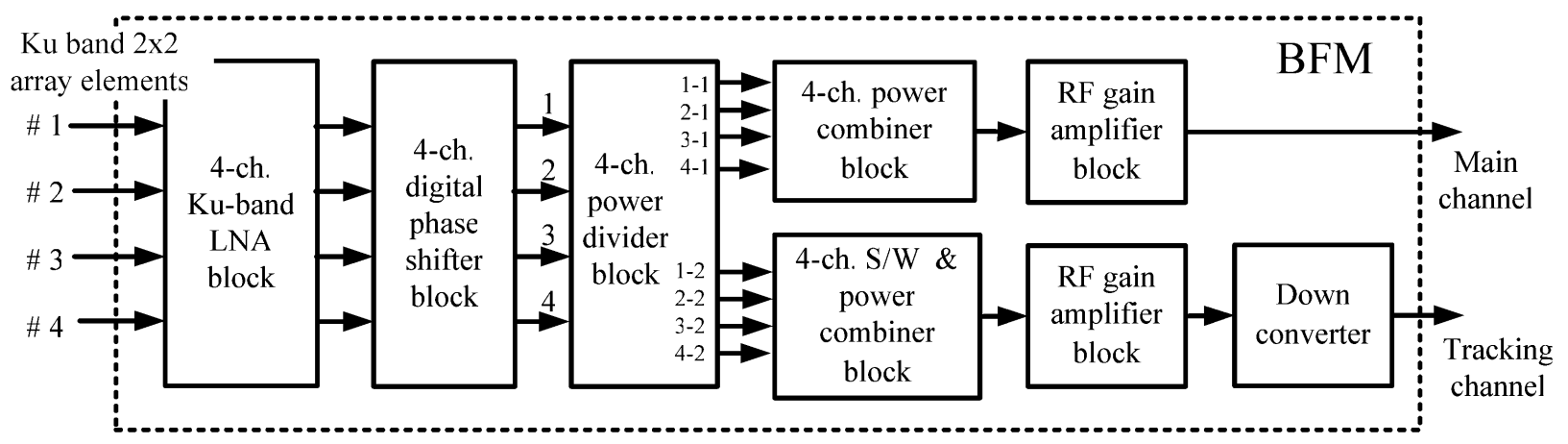

Fig. 6. Internal block diagram of the $K u$-band BFM.

\section{B. Ku-Band Beam Forming Module}

The BFM receives the $K u$-band satellite broadcasting signal by the $2 \times 2$ array elements of the tri-band feed, amplifies it, and forms both the main beam translated to the main channel as well as the tracking beam, which is implemented by electrical switching of the four squint partial beams (No. 1-4) and is then translated to the tracking channel in the STM. Fig. 6 shows the internal block diagram of the BFM. NEC's low noise device (NEC3210S01) is used in the 4-channel LNA, and Teltron's MMIC (TP002) is used as the 4-bit digital phase shifter element which is needed to calibrate the phase differences between four active channels. The switching unit (S/W in Fig. 6) is made by two cascade signal attenuators. Each signal attenuator is composed of a $90^{\circ}$ branch-line hybrid coupler and two switching diodes, and the measured channel switch isolation is more than $25 \mathrm{~dB}$. The output signal from the main channel goes through the rotary joint, and is then entered into the down-converter input. The output signal from the tracking channel goes to the tracking signal converter that transforms RF power to dc voltage; it also performs A/D conversion again in order to provide the digital data from the four squint beams to the antenna control unit.

The $K a$-band high-power signal by spatial coupling between the $K u$-band radiator and the $K a$-band dielectric rod is fed to the BFM input as a noise signal. The $K a$-band power coupled at the maximum EIRP is about $0 \mathrm{dBm}$. However, the reflection coefficient at the BFM input over the $K a$-band is close to one, and $\mathrm{Ku}$-band low noise devices are barely operated at the $\mathrm{Ka}$-band. Therefore, it is enough to insert a simple rejection filter with parallel open stubs in front of the LNA block to suppress the coupled $K a$-band signal. Such a simple stub filter has no effect on the $K u$-band performance.

In this paper, the measured noise figures of the main and tracking channel including input connector are less than 1.6 and $2.6 \mathrm{~dB}$, respectively, within the $K u$ operating band. The overall effective equivalent noise temperature, $T_{\text {eff Ku }}$ which includes an antenna noise temperature and is referenced at the BFM input, can be founded with taking into account the sky noise temperature $T_{\mathrm{SN}}$ of about $8 \mathrm{~K}$ in the $K u$-band at $45^{\circ}$ elevation angle [12], [13]. Loss $\alpha_{\mathrm{RAD}}$ in patch radiating elements has a value of about $0.2 \mathrm{~dB}, \operatorname{loss} \alpha_{\mathrm{FL}}$ in the coaxial feed line connecting the patch element and the BFM input is about $0.3 \mathrm{~dB}$. $T_{\mathrm{BFM}}$ is an equivalent noise temperature measured at the BFM

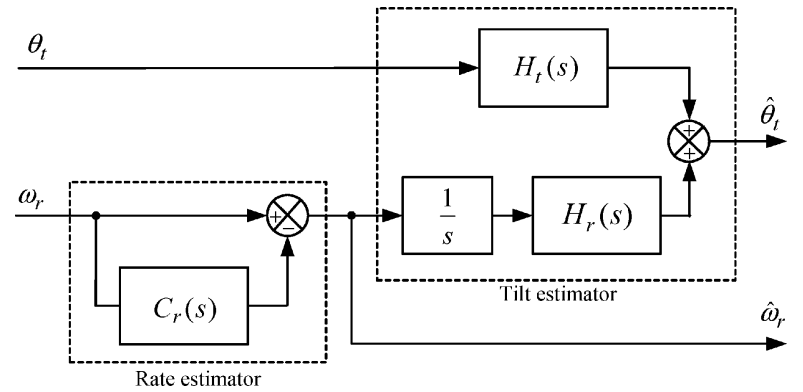

Fig. 7. Block diagram of the sensor signal processing.

input, its measured value in the main channel is about $129 \mathrm{~K}$. Therefore, the estimated system noise temperature at the BFM input is about $168 \mathrm{~K}$.

\section{Satellite Tracking Module}

The major function of the STM consists in providing a stable communication service by a permanent beam pointing to the target satellite under the movement of a vehicle. The STM consists of a sensor unit and a control unit. The sensor unit comprises a magnetic compass detecting the present azimuth of the antenna, and tilt and rate sensors detecting the present tilt angle and angular velocity, respectively, which involves the sensor signal processing algorithm for reliable sensor signal acquisition. The control unit performs the satellite tracking control on the basis of the current stabilizing data acquired from the sensors and the fine direction data from electrical tracking beams. Also, the control unit comprises motor drivers that enable beam pointing by a mechanical rotation of the antenna in azimuth and elevation.

1) Sensor Signal Processing: To ensure the precise satellite tracking, sensor accuracy is very important. Therefore, the sensors must be strong enough to resist the various motional frequencies of the vehicle's movement and the ambient temperature changes. Inexpensive generic sensors, used for the tile and rate measurements, are unsuitable for the satellite tracking control of a high gain antenna system with a narrow beamwidth. Tilt sensors are accurate in quasi-static conditions: that is, they are accurate in cases of low frequency angular motion but are inaccurate where high frequency movement is concerned. On the contrary, rate sensors show good accuracy at high frequencies of mechanical rotary motion. Furthermore, rate sensors may 


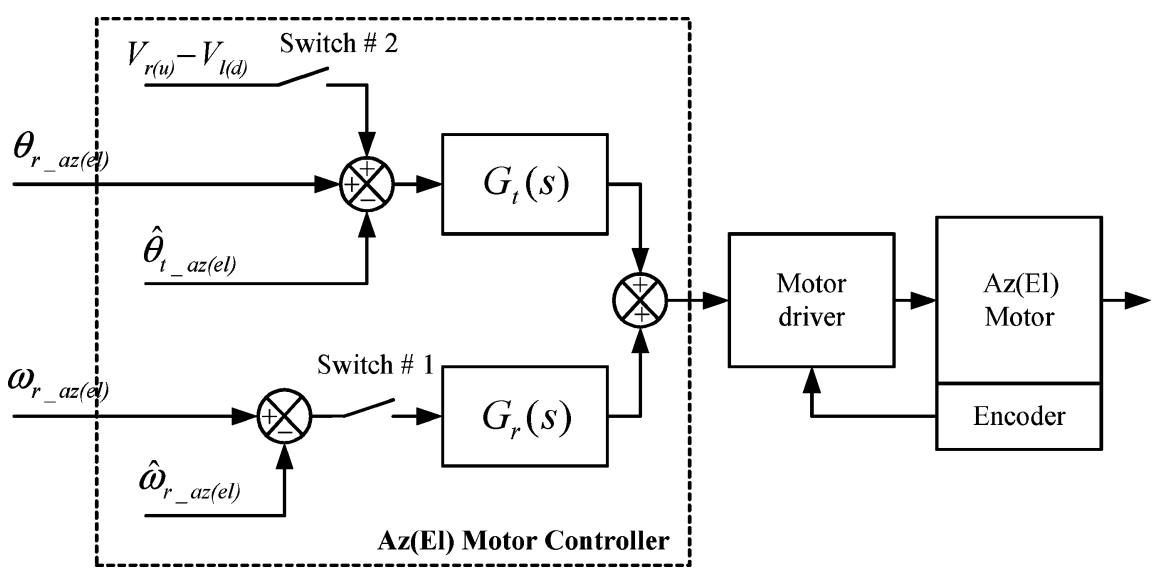

Fig. 8. Block diagram of motor control.

detect inaccurate angular velocity due to temperature drift, thus affecting the control performance of the mobile antenna system.

To overcome this problem, the specific signal processing is necessary. In this system, the ADXL203 of Analog Device and the ENV-05G of Murata are used as the inexpensive generic tilt and rate sensors, respectively. Fig. 7 shows a block diagram of the sensor signal processing involving the tilt and rate estimators, wherein, $\theta_{t}$ is the tilt angle output from the real tilt sensor, and $\hat{\theta}_{t}$ is the estimated tilt angle. Similarly, $\omega_{r}$ is the angular velocity output from the real rate sensor, and $\hat{\omega}_{r}$ is the estimated angular velocity. The relations can be given by the following transfer functions:

$$
\begin{aligned}
\hat{\omega}_{r}(s) & =\left[1-C_{r}(s)\right] \omega_{r}(s) \\
\hat{\theta}_{t}(s) & =H_{t}(s) \theta_{t}(s)+\frac{1}{s} H_{r}(s) \hat{\omega}_{r}(s) .
\end{aligned}
$$

In the rate estimator, the drift caused by ambient temperature change is eliminated. Generally, the ambient temperature has an ultra-low frequency characteristic which varies on a daily basis. Therefore, by extracting the temperature drift value and the initial bias value using the ultra-low frequency pass filter, $C_{r}(s)$, from the rate sensor output, it can be effectively eliminated.

As previously mentioned, the tilt sensor is accurate at low frequency while the rate sensor is accurate at high frequency. Therefore, utilizing such characteristics to estimate the tilt angle, the tilt sensor output is strongly contributed in a low frequency band, and the estimated rate is strongly done in a high frequency band. Here, $H_{t}(s)$ is the transfer function of a low pass filter, and $H_{r}(s)$ is that of a high pass filter. Additionally, they should have the same cutoff frequency and satisfy the relation $H_{t}(s)+H_{r}(s)=1$. The described sensor signal processor can be easily implemented with digital filters.

2) Satellite Tracking Control: The developed mobile antenna system tracks the target satellite with a mechanical control having two degrees of freedom in the azimuth and elevation angles from the help of the tilt and rate sensors and the squinted tracking beam which is formed by the BFM moves sequentially and periodically around the main beam. The satellite tracking flow is performed in three steps: a "search step" which searches for the target satellite until its signal has been detected; a "coarse tracking step" which uses signals coming from the tilt and rate sensors only after the satellite signal has been detected; and a "fine tracking step" which uses a signal from the electrically tracking beam. In the search and coarse tracking steps, the open-loop control from the satellite signal is carried out by a mechanical position control by the feedback of the tilt and rate sensors toward the reference azimuth and elevation angle of the target satellite. In the fine tracking as a final step, the closed-loop control with the satellite signal is performed by tracking beams that are sequentially and periodically switched by the BFM.

To enable beam pointing by a mechanical rotation of the antenna in azimuth and elevation, the block diagram of motor control is presented in Fig. 8. In the search step, the present location (latitude and longitude) of the mobile antenna system is given by GPS, and the longitude of the target satellite is given, hence, the target azimuth $\theta_{r_{-} a z}$ and the target elevation angle $\theta_{r_{-} e l}$ can be calculated and then set up. When the angles are set, the antenna is directed to the satellite by mechanical motion to detect the satellite signal. Once the satellite signal has been detected, the coarse tracking step is performed. And the switch \#1 is then turned on to receive the feedback of the angular velocities $\hat{\omega}_{r_{-} a z}$ and $\hat{\omega}_{r_{-} e l}$ for the azimuth and elevation angles respectively, in order to stabilize the antenna against the moving vehicle. Here, the target angular velocities, $\omega_{r_{-} a z}$ and $\omega_{r \_e l}$ are set at zero in order to keep the antenna motionless against the vehicle's motion. The $G_{t}(s)$ and $G_{r}(s)$ are the transfer functions of the PID controller. Finally, as the fine tracking step, the switch \#2 is additionally turned on to detect differences in the signal strength of the tracking beam in four sequential positions. The up/down beams signaling difference $V_{u}-V_{d}$ and the right/left beams signaling difference $V_{r}-V_{l}$ are fed-back for fine tracking. From the step by step, a continuous beam pointing to the target satellite can be achieved under the movement of a vehicle.

\section{TEST OF THE ANTENNA System}

The radiation pattern test of the fabricated antenna was performed using a test-jig and near-field measurement system in the anechoic chamber. The co- and cross-polarized radiation patterns measured at the central frequencies of the $\mathrm{Ka}$-band and $K$-band are shown in Figs. 9 and 10. Since the $K u$-band part of the antenna system consists of four active channels that are different from the $\mathrm{Ka} / \mathrm{K}$-band, initial phase compensation is required for four active channels. Therefore, the four partial patterns (beams) of the $K u$-band at the output of the BFM are 


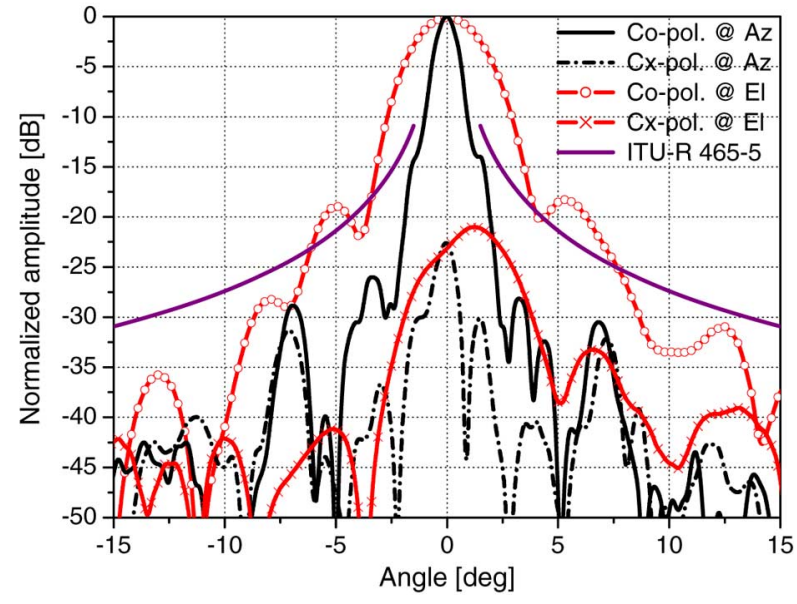

Fig. 9. Radiation patterns measured in the $K a$-band ( $f=30.485 \mathrm{GHz}$ ).

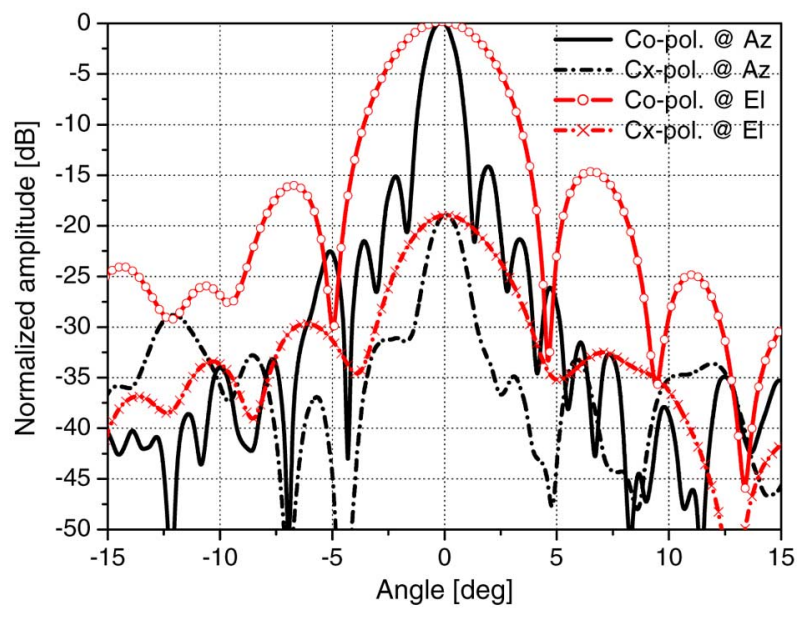

Fig. 10. Radiation patterns measured in the $K$-band ( $f=20.755 \mathrm{GHz}$ ).

measured. To form the main beam in the $K u$-band, which is the sum of four partial beams, phase compensation of $0^{\circ}, 90^{\circ}$, $180^{\circ},-90^{\circ}$ in the correspondent active channel is applied in order to match the phases of the partial beams in the far-field. Fig. 11 shows the measured main beam radiation patterns after the phase compensation, as well as the tracking beams. From Fig. 11, the left and right tracking beams are squinted about $\pm 1^{\circ}$ and its scan loss is $0.5 \mathrm{~dB}$. And also the upper and down tracking beams are squinted about $\pm 2^{\circ}$ and its scan loss is $2.0 \mathrm{~dB}$. The major electrical performances of the tri-band radiation patterns measured at each center frequency shown in Figs. 9-11 are summarized in Table III. The values of antenna gain and efficiency presented in Table III include feed cable losses.

The whole antenna system after assembly is shown in Fig. 12. Fig. 13 shows a schematic diagram of the satellite link test with the antenna system mounted on a mobile simulator vehicle. The measurement of $(G / T)_{\mathrm{AUT}}$, which represents the receiving sensitivity of the antenna under testing (AUT), is carried out with the $K a$-band reception signal and the $K u$-band broadcasting signal from Koreasat-3, using a reference standard parabolic dish antenna for which the $(G / T)_{\text {DISH }}$ characteristics are known. For this purpose, the $(C / N)_{\text {DISH }}$ of the reception signal is measured with a standard dish antenna,

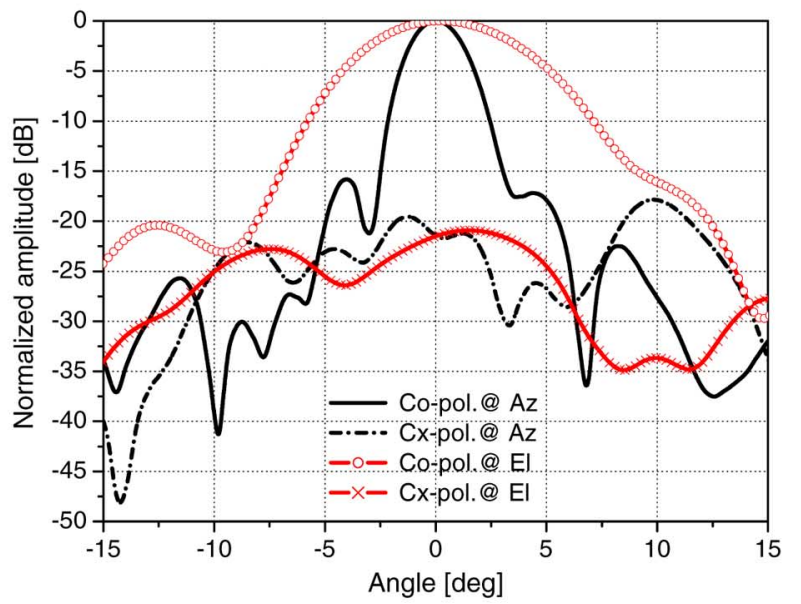

(a)

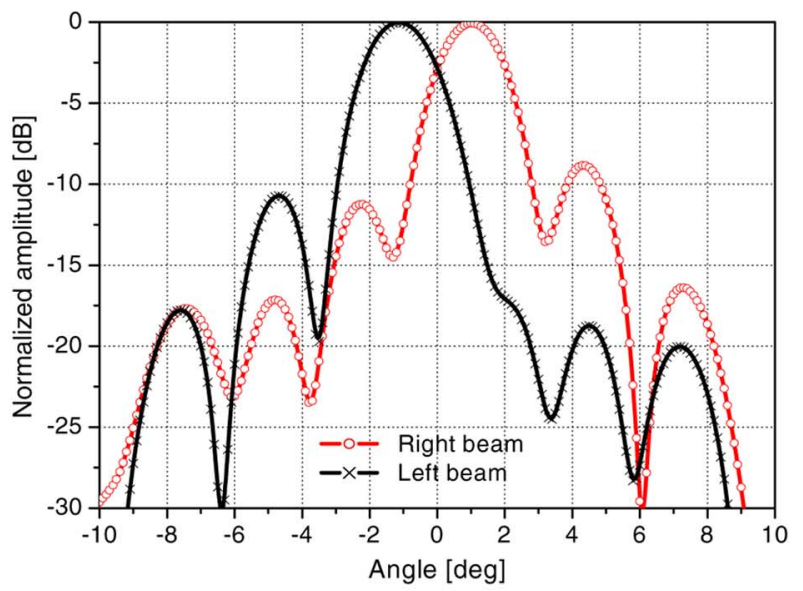

(b)

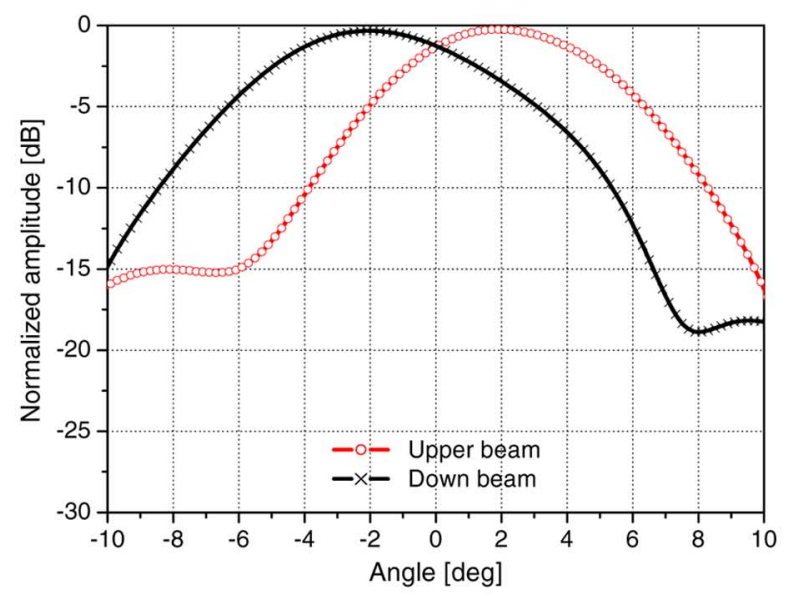

(c)

Fig. 11. Radiation patterns measured at the $K u$ band ( $\mathrm{f}=11.85 \mathrm{GHz}$ ): (a) main beam patterns, (b) left and right tracking beam patterns, and (c) upper and down tracking beam patterns.

and $(C / N)_{\mathrm{AUT}}$ of the antenna under testing is measured too. Therefore, the measured $(G / T)_{\text {AUT }}$ of the antenna under testing can be obtained comparatively from the formula (6) [14]

$$
\left(\frac{G}{T}\right)_{\mathrm{AUT}}=\left(\frac{C}{N}\right)_{\mathrm{AUT}}-\left(\frac{C}{N}\right)_{\mathrm{DISH}}+\left(\frac{G}{T}\right)_{\mathrm{DISH}} .
$$


TABLE III

Main Electrical Performances of the Measured Tri-BAnd Radiation Patterns and Gains

\begin{tabular}{|c|c|c|c|c|c|}
\hline Frequency band & Gain & Efficiency & Beam width & $\begin{array}{c}\text { Side-lobe } \\
\text { level }\end{array}$ & $\begin{array}{c}\text { Cross-polarization } \\
\text { @ bore-site }\end{array}$ \\
\hline$K a$-band & $39.6 \mathrm{dBi}$ & 0.54 & $1.0^{\circ} \times 3.3^{\circ}$ & $13.9 \mathrm{dBc}$ & $22.6 \mathrm{dBc}$ \\
\hline$K$-band & $36.5 \mathrm{dBi}$ & 0.57 & $1.3^{\circ} \times 4.0^{\circ}$ & $14.1 \mathrm{dBc}$ & $18.9 \mathrm{dBc}$ \\
\hline$K u$-band & $29.3 \mathrm{dBi}$ & 0.34 & $2.6^{\circ} \times 6.4^{\circ}$ & $15.8 \mathrm{dBc}$ & $21.4 \mathrm{dBc}$ \\
\hline
\end{tabular}

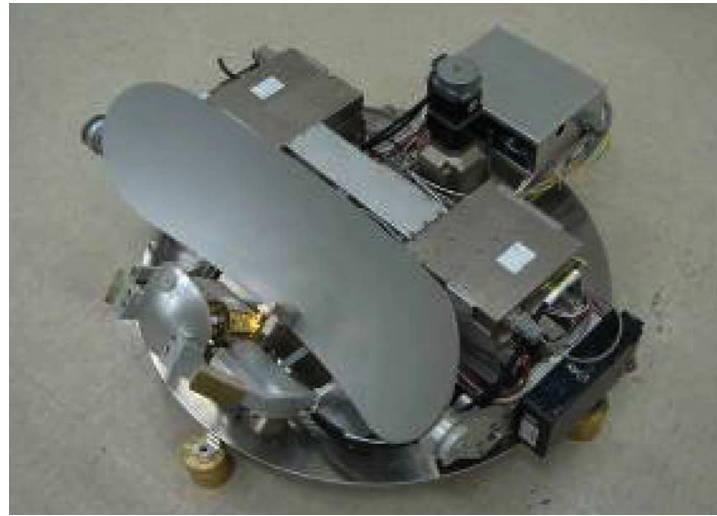

Fig. 12. Photo of the entire assembled mobile antenna system.

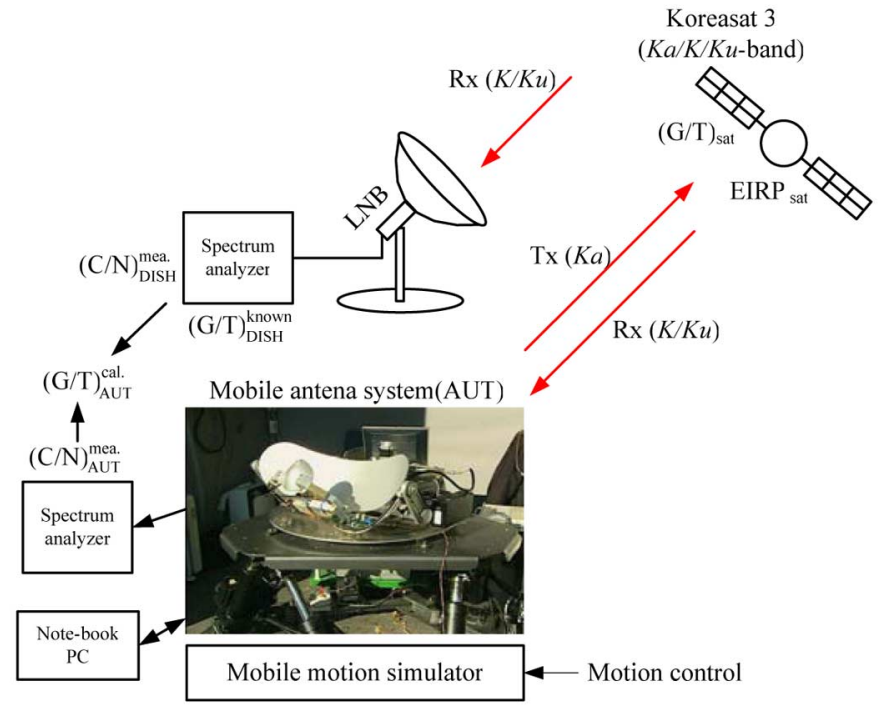

Fig. 13. Satellite link test configuration using mobile motion simulator.

The measurements were performed under stable conditions, so the measured results do not include the antenna gain degradation caused by errors of tracking. The measured receiving sensitivity showed more than $7.1 \mathrm{~dB} / \mathrm{K}$ in the $K u$-band (the main channel) and more than $12.2 \mathrm{~dB} / \mathrm{K}$ in the $K$-band. The system $G / T$ estimation using the measured antenna gain and noise figure data directly measured from the $\mathrm{Ku}$-band BFM gives the value of 7.0 $\mathrm{dB} / \mathrm{K}$ at the elevation angle of $45^{\circ}$. Similarly, in the case of the $K$-band, it gives the value of $12.1 \mathrm{~dB} / \mathrm{K}$ at the same elevation angle. These estimated performances correspond well with the measured ones. The system $G / T$ measured in $K u$-band do not meet with the initial specification, but it is acceptable for a stable TV reception.

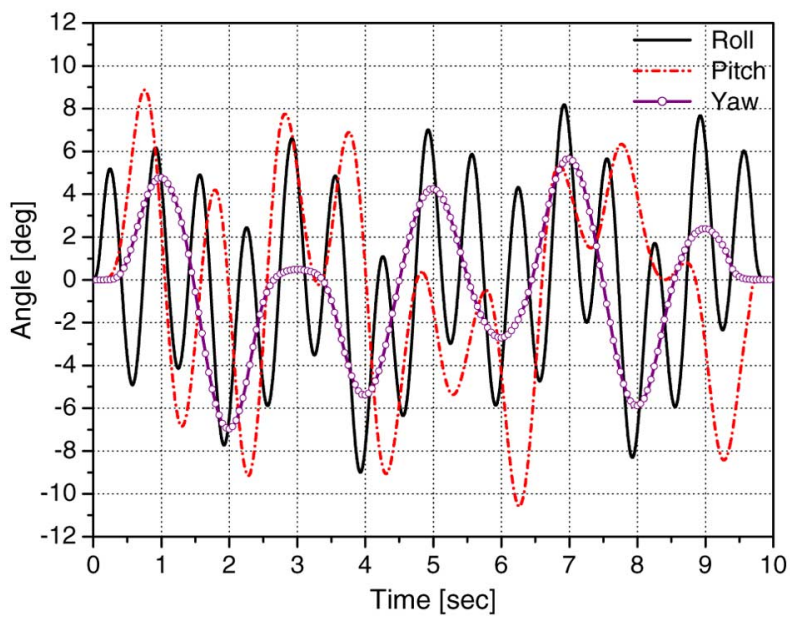

Fig. 14. Shipboard test conditions generated by mobile motion simulator.

The performances of the Tx EIRP and satellite tracking tests of the antenna system were performed with the system mounted on a mobile motion simulator and Koreasat-3. The maximum EIRP was measured in a similar manner to that of the $\operatorname{Rx} G / T$ : by a relative method using a reference of a fixed transmission terminal antenna with a known Tx EIRP value. The maximum EIRP was measured to be $43.7 \mathrm{dBW}$ at $30.085 \mathrm{GHz}$. The satellite tracking performance test was carried out with the system mounted on the mobile simulator and linked directly to Koreasat-3. In the shipboard mobile testing conditions for rolling, pitching and yaw, as shown in Fig. 14, the satellite tracking performance showed a tracking loss of less than $0.5 \mathrm{~dB}$ within a tracking error range of $\pm 0.2^{\circ}$.

\section{SATEllite Link Test of The ANTENNA System}

After verifying the tracking performance using the mobile motion simulator in the outdoor laboratory, a satellite access test in the actual field was performed with the antenna system mounted on a real shipboard as shown in Fig. 15, and a test car as shown in Fig. 16. Fig. 17(a) represents the test results when the antenna system was installed on a large test ship of the 3,000-ton class in the South Sea of Korea. Here, the tracking loss is excellent at less than $0.5 \mathrm{~dB}$. Since the satellite signal is not blocked at sea, the tracking performance must be deemed good and stable. Fig. 17(b) corresponds to the case whereby the mobile antenna system is mounted and tested on a van driven at $100 \mathrm{~km} / \mathrm{h}$ on the highway. Satellite tracking is stable with a tracking loss of less than $1 \mathrm{~dB}$. In the graph, the section between the 23rd and 24th minutes corresponds to a tunnel in which the signal could not be detected and only the noise level can be seen. 


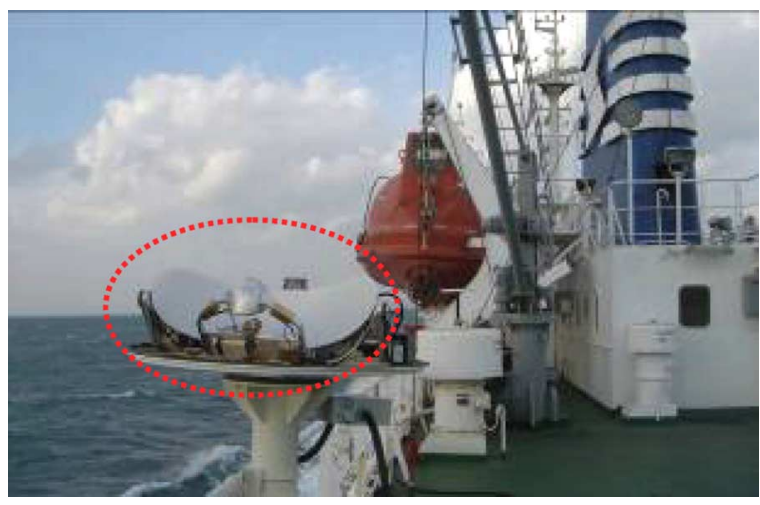

Fig. 15. Antenna system installed on a large test ship.

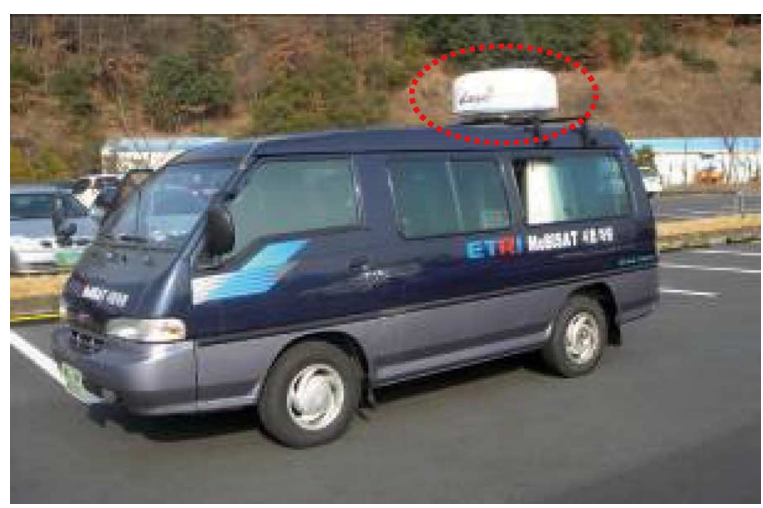

Fig. 16. Antenna system installed on the test van.

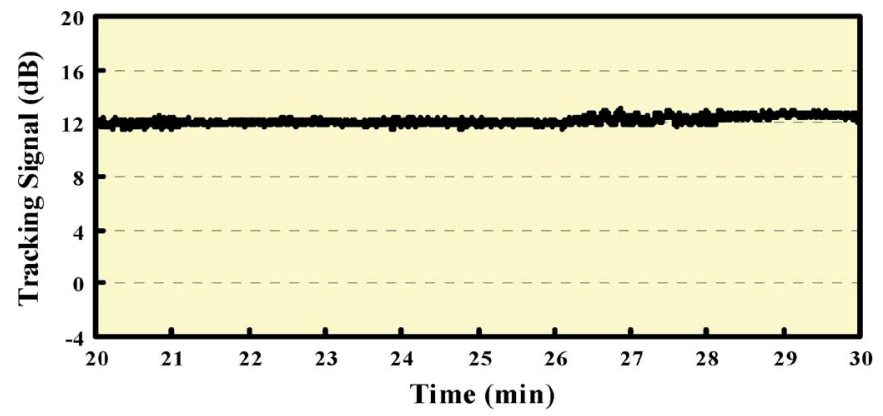

(a)

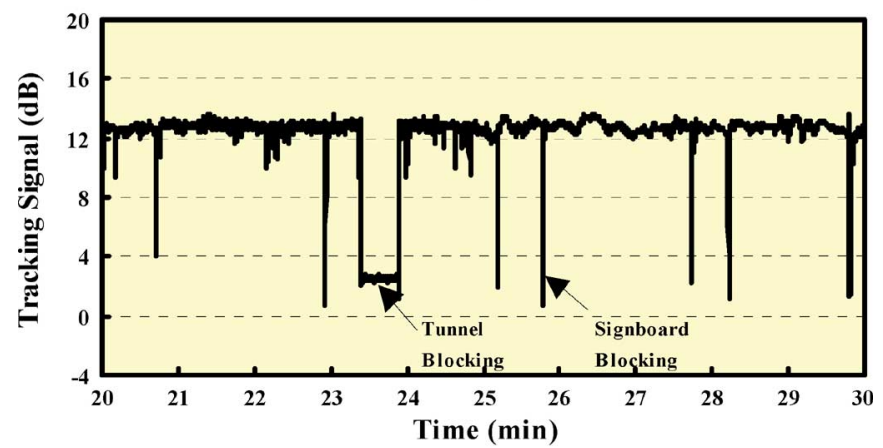

(b)

Fig. 17. Satellite link test results: (a) test results using the large ship and (b) test results using the test van.

However, the satellite was detected and tracked again as soon as the vehicle exited the tunnel. The short cutoffs of the signal are blocks caused by traffic signs.

\section{CONCLUSION}

A tri-band mobile antenna system which operates by utilizing the geo-stationary satellite Koreasat- 3 in tri-band $(K a, K$, and $K u)$ was designed, and a pilot product was fabricated and tested. From the fabricated antenna system, the measured $K a$-band EIRP was more than $43.7 \mathrm{dBW}$, and the receiving $G / T$ sensitivities measured in the $K$-band and $K u$-band were more than $12.2 \mathrm{~dB} / \mathrm{K}$ and $7.1 \mathrm{~dB} / \mathrm{K}$, respectively. These test results showed that forward link of $10 \mathrm{Mbps}$ data reception and return link of $1 \mathrm{Mbps}$ data transmission can be implemented via Koreasat-3.

Since the tri-band antenna system design technology permits the provision of diverse broadband services including super high-speed interactive internet access, video communication, video on demand, and high quality satellite broadcasting, its technical outcome could be utilized as a core infrastructure technology for the next-generation ubiquitous network that will link satellite and wireless networks in the future.

\section{ACKNOWLEDGMENT}

The authors are greatly indebted to I. L. Vilenko, Dr. Z. A. Yanykian, Dr. Y. B. Korchemkin, Dr. A. S. Reutov, A. E. Kazaryan and S. W. Ryu for their contributions to the design and testing of some of the components of the antenna system.

\section{REFERENCES}

[1] V. Schena and F. Ceprani, "FIFTH project solutions demonstrating new satellite broadband communication system for high speed train," in Vehicular Technology Conf., May 2004, vol. 5, pp. 2831-2835.

[2] A. Monk et al., "An ultra-low profile airborne reflector antenna subsystem for broadband satellite communications," presented at the 21st AIAA Int. Communications Satellite Systems Conf. and Exhibit AIAA 2003-2316, Yokohama, Japan, Apr. 15-19, 2003.

[3] A. Densmore and V. Jamnejad, "A satellite-tracking K- and Ka-band mobile vehicle antenna system," IEEE Trans. Veh. Technol., vol. 42, no. 4, pp. 502-513, Nov. 1993.

[4] Y. Inasawa, S. Kuroda, K. Kusakabe, I. Naito, and Y. Konishi, "Aeronautical ultra-low-profile reflector antenna designed by physical optics shaping technique," presented at the IEICE Society Conf., C-1-5, (in Japanese) Tokushima, Japan, Sep. 21-24, 2004.

[5] H. Wakana et al., "COMETS for Ka-band and millimeter-wave advanced mobile satellite communications and $21 \mathrm{GHz}$ advanced satellite broadcasting experiments," in Proc. IEEE Int. Conf. on Communications, Jun. 1998, vol. 1, pp. 79-83.

[6] G. J. Hawkins, D. J. Edwards, and J. P. McGeehan, "Tracking systems for satellite communications," Proc. Inst. Elect. Eng., vol. 135, no. 5, pp. 393-407, Oct. 1988.

[7] B. Y. Kinber, S. N. Kaptsov, and A. V. Shishlov, "Inverse problems in optics and antenna theory," in Lectures of the IX USSR's School on Diffraction and Propagation (in Russian), Chistopol, 1988, p. 117.

[8] A. S. Reutov and A. V. Shishlov, "Features of the stepwise contour beam reflector antenna synthesis using cubic spline representation of the reflector surface," (in Russian) Electromagn. Waves and Elect. Syst. Moscow, no. 2, pp. 4-14, 2003.

[9] Reference Earth-Station Radiation Pattern for Use in Coordination and Interference Assessment in the Frequency Range From 2 to About 30 $\mathrm{GHz}$, Recommendation ITU-R S.465-5.

[10] T. Hori and N. Nakajima, "Broadband circularly polarized microstrip array antenna with coplanar feed," Electron. Commun. Jpn., vol. 69, no. 11, pt. 1, pp. 76-83, 1986.

[11] S. Y. Eom and Y. B. Korchemkin, "A new comb circular polarizer suitable for millimeter-band application," ETRI J.l, vol. 28, no. 5, pp. 656-659, Oct. 2006.

[12] M. I. Skolnik, Radar Handbook. New York: McGraw-Hill, 1991, pp. 2.26-2.31.

[13] L. V. Blake, "Antenna and receiving system noise-temperature calculation," Proc. IEEE, vol. 49, pp. 1568-1569, Oct. 1961. 
[14] S. Y. Eom et al., "Design and test of RACM in a Ku-band vehicular APAA system for DBS," in Proc Int. Conf. on Electromagnetics in Advanced Applications, Sep. 1999, pp. 215-218.

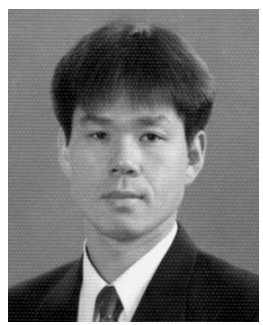

Soon Young Eom (M'01) was born in Gangwon-do, Korea, on May 2, 1964. He received the B.S. and M.S. degrees in electronic engineering from Yonsei University, Seoul, Korea, in 1988 and 1990, respectively, and the Ph.D. degree in electronic engineering from Yonsei University, Seoul, Korea, in 2003.

Since 1990, he has worked at the Electronics and Telecommunications Research Institute (ETRI), Daejeon, Korea, as a Principal member of research staff. From 1991 to 1993, he participated in the joint project with Alenia Spazio S.P.A. in Italy, for developing the very small aperture terminal (VSAT) system. With a study on flat-topped radiation pattern using a multilayered disk array structure, he received the Ph.D. degree. From 1996 to 2005, he took part in developing many mobile active phased array antenna systems for direct broadcasting satellite (DBS) reception and satellite communications. He is now involved to develop the reconfigurable antenna and its system application technology. His research interests include active phased array antenna systems, microwave circuits and systems, and advanced mobile base station antenna systems.

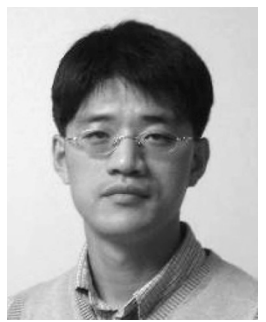

Seong Ho Son was born in Pusan, Korea, on April 16, 1974. He received the B.S. degree in control and mechanical engineering from Pusan National University, in 1997, and the M.S. degree in mechanical engineering from Pohang University of Science and Technology (POSTECH), Pohang, Korea, in 1999. He is currently working toward the Ph.D. degree in the Department of Mechanical Engineering at POSTECH.

From 1999 to 2001, he was with the Technical Research Center of Daewoo Motors. Since 2001, he has been with Electronics and Telecommunications Research Institute (ETRI), Daejeon, Korea, as a Senior Member of Research Staff, where he has been engaged in research on mobile antenna systems for satellite communications. His research interests are in phased array antenna systems, array design and optimization, mechatronics, and load-bearing antenna structures.

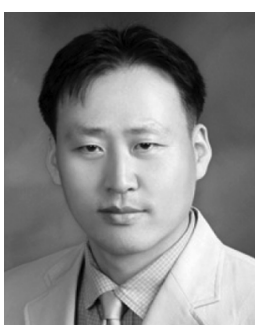

Young Bae Jung was born in Seoul, Korea, in October 1971. He received the B.S. degree in radio science and engineering from Kwangwoon University at Seoul, Korea, in 1999 and the M.S. degree in electronic engineering from the Information and Communication University (ICU), Daejeon, Korea, in 2001, where he is currently working toward the Ph.D. degree.

Since 2001, he has been with the Electronics and Telecommunications Research Institute (ETRI), Daejeon, Korea, as a Senior Researcher. His work is focused on the active phased array antenna systems and his research interests include array antenna system and active/passive components in the area of microwave engineering.

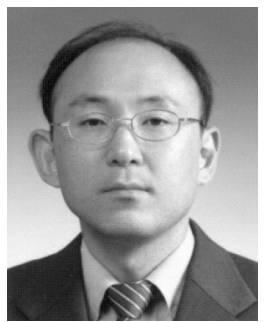

Soon Ik Jeon received the B..S and M.S. degrees in electronic engineering from Korea University, Seoul, Korea, in 1984 and 1996, respectively, and the Ph.D. degree in electronic engineering from Chung-Nam University, Daejeon, Korea, in 2003.

From 1984 to 1990, he worked at Samsung Electronics, Seoul, as a member of research staff. Since 1990, he has worked at the Electronics and Telecommunications Research Institute (ETRI), Daejeon, Korea. He is the Team Leader of the Antenna Technology Research Team, Advanced
Radio Technology Research Group, Radio and Broadcasting Research Division, ETRI. He was involved in the development of the mobile active phased array antenna systems for direct broadcasting satellite (DBS) reception and satellite communications. He is now involved to develop the reconfigurable antenna and its system application technology and the microwave tomography for breast tumor detection based on EM technology. His research interests include antennas and active phased array antennas and their applications for communications and biomedical sensing.

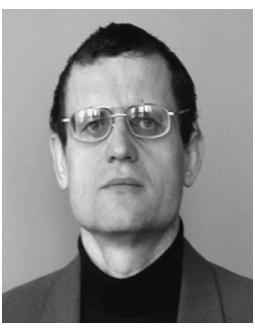

Sergei A. Ganin was born in Moscow, Russia, in 1955. He received the M.S. degree in physics from the Moscow States University, in 1979.

From 1979, he worked on wave propagation theory in the Institute of Radioengineering and Electronics of the Russian Academy of Science. Since 1986, he has worked as a Senior Research Engineer on antenna theory and technology in the Research Institute of Radio Physics (now the Joint-Stock Company (JSC) Radiophyzika), Moscow, Russia. His research interests include technology, theory and numerical analysis of radiating structures and phased array antennas.

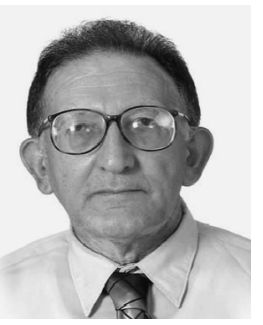

Anatoly G. Shubov received the M.S. degree in radio engineering from the Moscow Power Engineering Institute, Moscow, Russia, in 1953 and the Ph.D. degree in antennas and microwave engineering from the Research Institute of Long Distance Radiocommunications (RILDRC), Moscow, in 1973.

From 1953 to 1976, he was with the Antenna Department of RILDRC. Since 1976, he has been with the Antenna and Microwave Engineering Division of the Research Institute of Radio Physics (now the Joint-Stock Company (JSC) Radiophyzika), Moscow, Russia, working as a Leading Research Associate. Since 1993, he has been with the JSC "Apex". He took part in a number of projects on reflector and lens antennas as well as phased array antennas. The main area of his research interest is array antenna technology.

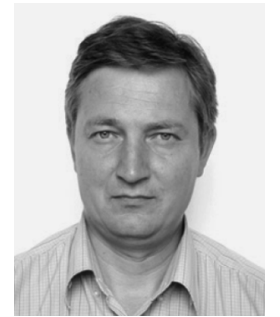

Alexey K. Tobolev was born in Kharkov, Ukraine, in 1956. He received the M.S. and Ph.D. degrees in antennas and microwave engineering from the Moscow Institute of Physics and Technology (MIPT), Moscow, Russia, in 1979 and 1986, respectively.

Since 1979, he has worked with the Research Institute of Radio Physics (now the Joint-Stock Company (JSC) Radiophyzika, Moscow, Russia) as a Research Engineer, and since 1986, as a Team Leader in Antenna Laboratory. Since 1992, he has been with JSC Apex serving as a Project Manager in various antenna developments. His research interests include theory and technology of reflector antennas, horn antennas, hybrid antennas and RF measurements.

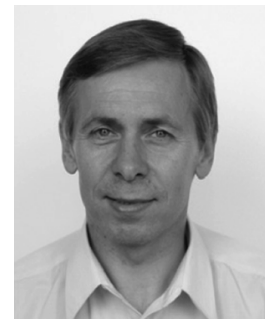

Alexander V. Shishlov (M'99) received the M.S. degree in radioelectronics and the Ph.D. degree in antenna theory and technology from the Moscow Institute of Physics and Technology, Moscow, Russia, in 1977 and 1983, respectively.

Since 1977, he was worked on antenna theory and technology at the Research Institute of Radio Physics (now the Joint-Stock Company (JSC) Radiophyzika), Moscow, Russia. Since 1992, he served as Project Leader of various antenna developments in the Joint-Stock Company (JSC) Radiophyzika, Moscow, Russia and JSC "Apex". His research interests include theory and technology of quasi-optic antennas and phase array antennas. 